\title{
Anatomy of the extraneural blood supply to the intracranial oculomotor nerve
}

Institute of Ophthalmology, University College Dublin, Mater Misericordiae

Hospital, Dublin, Ireland

M Cahill

P Eustace

\section{Department of}

Anatomy, The Medical School, University College Dublin, Dublin, Ireland M Cahill

J Bannigan

Correspondence to: Professor Peter Eustace, Institute of Ophthalmology, University College Dublin, Mater Misericordiae Hospital, 60 Eccles Street, Dublin 7, Ireland.

Accepted for publication 11 October 1995

\begin{abstract}
Aims-An anatomical study was undertaken to determine the extraneural blood supply to the intracranial oculomotor nerve.

Methods-Human tissue blocks containing brainstem, cranial nerves II-VI, body of sphenoid, and associated cavernous sinuses were obtained, injected with contrast material, and dissected using a stereoscopic microscope.

Results-Eleven oculomotor nerves were dissected, the intracranial part being divided into proximal, middle, and distal (intracavernous) parts. The proximal part of the intracranial oculomotor nerve received extraneural nutrient arterioles from thalamoperforating arteries in all specimens and in six nerves this blood supply was supplemented by branches from other brainstem vessels. Four nerves were seen to be penetrated by branches of brainstem vessels and these penetrating arteries also supplied nutrient arterioles. The middle part of the intracranial oculomotor nerve did not receive nutrient arterioles from adjacent arteries. The distal part of the intracranial oculomotor nerve received nutrient arterioles from the inferior cavernous sinus artery in all 11 nerves and in seven nerves this was supplemented by a tentorial artery arising from the meningohypophyseal trunk. The inferior hypophyseal artery arose from the meningohypophyseal trunk in all 11 cavernous sinuses dissected.

Conclusion-This study shows a constant pattern to the blood supply of the intracranial oculomotor nerve. It also highlights the close relation between the blood supplies to the intracavernous oculomotor nerve and the pituitary gland.

(Br f Ophthalmol 1996; 80: 177-181)
\end{abstract}

Up to now no definitive detailed study of the blood supply to the intracranial portion of the third cranial nerve has been published. It is this portion of the third cranial nerve that has particular importance in neuro-ophthalmology. Bartholdy, in 1897, first described that cranial nerves received nutrient vessels from adjacent arteries. ${ }^{1}$ However, technical difficulties prevented any definite anatomical patterns being demonstrated. It was not until 1957 that Dreyfus et al, in a paper dealing with diabetic ophthalmoplegia, demonstrated that arteries from the basilar circulation and circle of Willis gave off nutrient arterioles to the intracranial part of the oculomotor nerve. ${ }^{2}$ These vessels have since been classified as extraneural vessels as they arise from outside the nerve and ramify on the surface of the nerve. In 1965, Parkinson attempted to demonstrate and name the branches of the intracavernous internal carotid artery. ${ }^{3}$ One of these branches, the newly titled meningohypophyseal trunk was seen to provide a nutrient arteriole to the oculomotor nerve in the majority of dissections. The inferior hypophyseal artery was also seen to arise from the meningohypophyseal trunk. Seven years earlier McConnell had demonstrated that this vessel provided a large portion of the pituitary blood supply. ${ }^{4}$

Asbury and colleagues provided further anatomical detail in $1970 .^{5}$ They set out to explain the clinical findings of diabetic ophthalmoplegia on a pathological basis. Using a serial section technique, they demonstrated an intraneural network of small arterioles within the oculomotor nerve. By this technique it was also determined that the extraneural arterioles to the oculomotor nerve arose proximally from a variety of brainstem arteries and distally from non-specific branches of the inferior cavernous sinus artery. More recently, three studies have set out to demonstrate the anatomy of the extraneural blood supply to the proximal part (also termed the interpeduncular or cisternal part) of the intracranial oculomotor nerve. ${ }^{6-8}$ Two of these studies by Milisavljevic et $a l,{ }^{8}$ and Marinkovic and Gibo, ${ }^{7}$ noted that the proximal intracranial oculomotor nerve can be penetrated by branches of brainstem arteries. These penetrating arteries can also supply nutrient arterioles to the nerves they penetrate.

The present anatomical study was undertaken to demonstrate the extraneural blood supply to the intracranial oculomotor nerve. We wanted to clarify the anatomical details using information from earlier studies ${ }^{1-8} 1^{10-12}$ and our own dissection findings (Fig 1). We also examined the relation between the blood supply to the intracavernous section of the oculomotor nerve and that to the pituitary gland.

\section{Materials and methods}

Six tissue blocks containing midbrain, pons, cerebellum, cranial nerves II-VI, basilar artery (and branches), body of sphenoid with associated pituitary gland, and cavernous sinuses were removed from postmortem subjects and immediately fixed in a solution of $10 \%$ formalin. To facilitate identification of vessels supplying the intracranial oculomotor nerve and other structures, the basilar and internal carotid arteries were injected with India ink before dissection. The specimens were dis- 


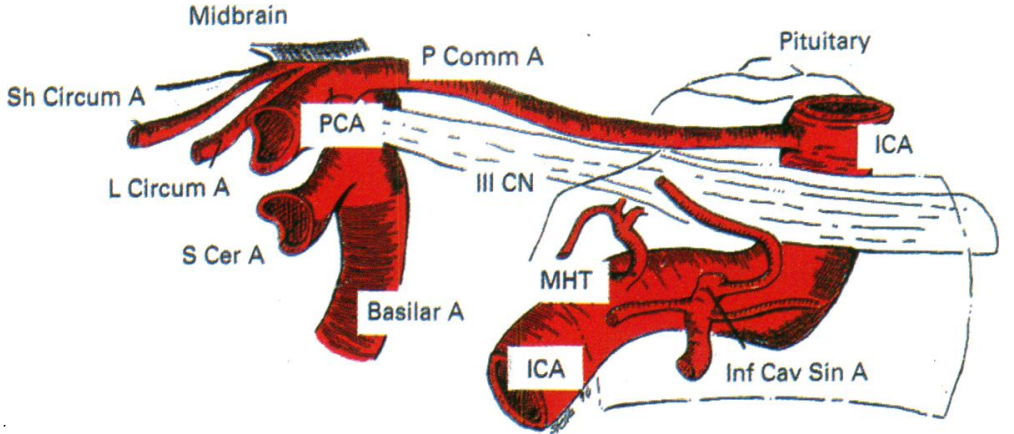

Figure 1 The blood supply to a right intracranial oculomotor nerve seen from the right. $P C A=$ posterior cerebral artery, $S$ Cer $A=$ superior cerebellar artery, $L$ Circum $A=$ long circumflex artery, Sh Circum $A=$ short circumflex artery, $P$ Comm $A=$ posterior communicating artery, III $C N=$ oculomotor nerve, ICA=internal carotid artery, $M H T=$ meningohypophyseal trunk, Inf Cav Sin $A=$ inferior cavernous sinus artery. Dark outline represents the limits of the cavernous sinus.

sected with the aid of a stereoscopic microscope. Photographs were taken and drawings were made at all stages of the dissections.

\section{Results}

Our of 12 possible intracranial oculomotor nerves, 11 were dissected. For simplicity the intracranial oculomotor nerve was anatomically divided into three parts. The proximal part consisted of the part from the emergence of the nerve at the cerebral peduncles to a point just distal to the anastomosis of the posterior cerebral and posterior communicating arteries. The middle part was that part from the latter point to just before the oculomotor nerve enters the cavernous sinus. The distal part was that part of the nerve within the cavernous sinus.

PROXIMAL NERVE

In all specimens, the proximal parts of the oculomotor nerve were supplied by thalamoperforating arteries arising from the posterior cerebral artery (Figs 2A and 2B). In five oculomotor nerves these were the only extraneural nutrient vessels to the proximal nerve. In the remaining six nerves these were supplemented by a number of brainstem arteries, outlined in Table 1 . The proximal part of the intracranial oculomotor nerve was seen to be penetrated by arteries in four nerves, which also supplied nutrient vessels to the nerve. In two nerves the penetrating artery was the long circumflex artery, in one nerve it was a thalamoperforating artery, and both a thalamoperforating artery

Table 1 Outline of brainstem vessels which provided supplementary nutrient arterioles to proximal part of intracranial oculomotor nerve

\begin{tabular}{lllll}
\hline Specimen & Artery & & & \\
\cline { 2 - 5 } No & PCA & PComm $A$ & $S$ Cer $A$ & LCircum $A$ \\
\hline 6 & & & $\checkmark$ \\
7 & & & $\checkmark$ \\
8 & & & \\
9 & & $\checkmark$ & & \\
10 & $\checkmark$ & $\checkmark$ & $\checkmark$ & $\checkmark$
\end{tabular}

$\mathrm{PCA}=$ posterior cerebral artery, $\mathrm{P}$ Comm $\mathrm{A}=$ posterior communicating artery, $S$ Cer $A=$ superior cerebellar artery, $\mathrm{L}$ Circum $\mathrm{A}=$ long circumflex artery. and a long circumflex artery penetrated the remaining nerve.

\section{MIDDLE NERVE}

The middle part of the intracranial oculomotor nerve was not seen to receive nutrient arterioles along its length from adjacent arteries.

DISTAL NERVE

In seven of 11 cavernous sinuses examined, the oculomotor nerve and its surrounding tentorium was seen to receive nutrient arterioles from an anteriorly directed tentorial artery which was a branch of the meningohypophyseal trunk (Figs 3A and 3B). In all 11 cavernous sinuses examined, an attempt was made to identify four branches of the inferior cavernous sinus artery - an anterior branch, a tentorial branch, a descending branch, and a posterior branch (Figs 4A and 4B). All 11 distal oculomotor nerves received nutrient arterioles from branches of the inferior cavernous sinus artery. In four nerves these nutrient arterioles arose from the anterior branch, in five they arose from the tentorial branch and in two nerves both the anterior branch and the tentorial branch supplied nutrient vessels to the oculomotor nerve.

In all 11 of the cavernous sinuses dissected the inferior hypophyseal artery was seen to arise from the meningohypophyseal trunk (Figs 3A and 3B). The ophthalmic artery did not supply nutrient arterioles to the oculomotor nerve in any of the 11 cavernous sinuses dissected.

\section{Discussion}

To date, no study has attempted systematically to identify and name the extraneural blood supply to the intracranial oculomotor nerve. The term extraneural is used to encompass those small arterioles arising from larger arteries adjacent to the oculomotor nerve. These are distinct from the intraneural arterioles which form a plexus of capillaries within the nerve substance. We felt that the clearest way to demonstrate the anatomy of the extraneural blood supply to the oculomotor nerve would be to divide it into three. Those segments of the nerve that make up these divisions (proximal, middle, and distal) have already been outlined in the results section.

Two early studies using postmortem subjects combined with anatomical dissections are important. ${ }^{25}$ These were undertaken to determine the pathological basis of diabetic ophthalmoplegia and demonstrated some of the extraneural nutrient arterioles to the proximal part of the intracranial oculomotor nerve. The first, by Dreyfus et $a l^{2}$ demonstrated that the extraneural nutrient arterioles could arise from the posterior cerebral and superior cerebellar arteries. Unfortunately, the reporting of these dissections was unsystematic. The second study by Asbury and colleagues, ${ }^{5}$ systematically determined the intraneural blood supply to the proximal part of the intracranial 


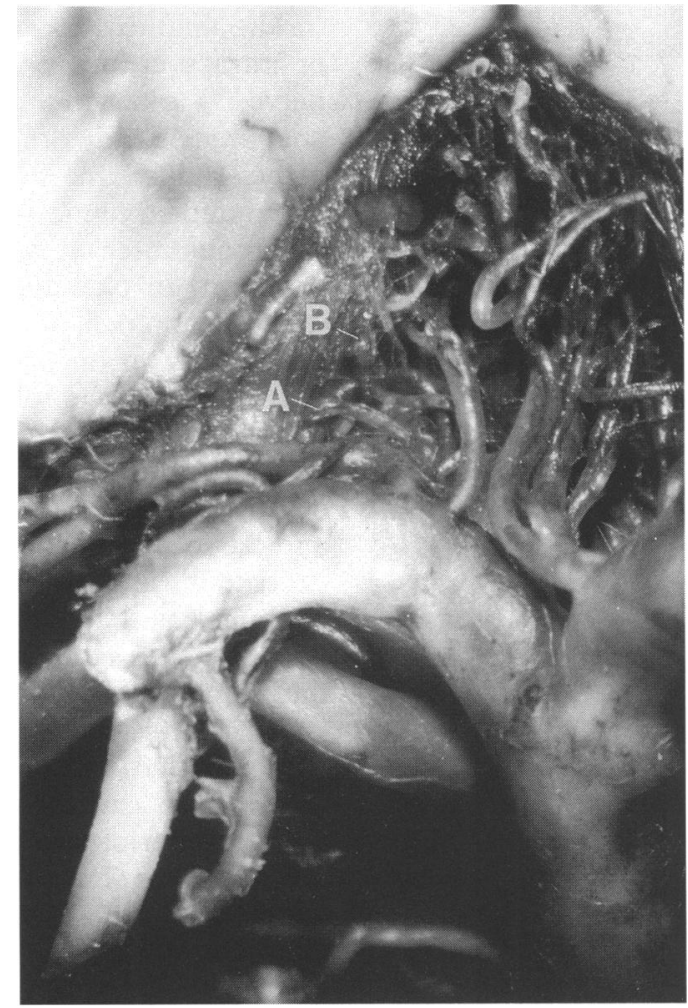

Fig 2A

B

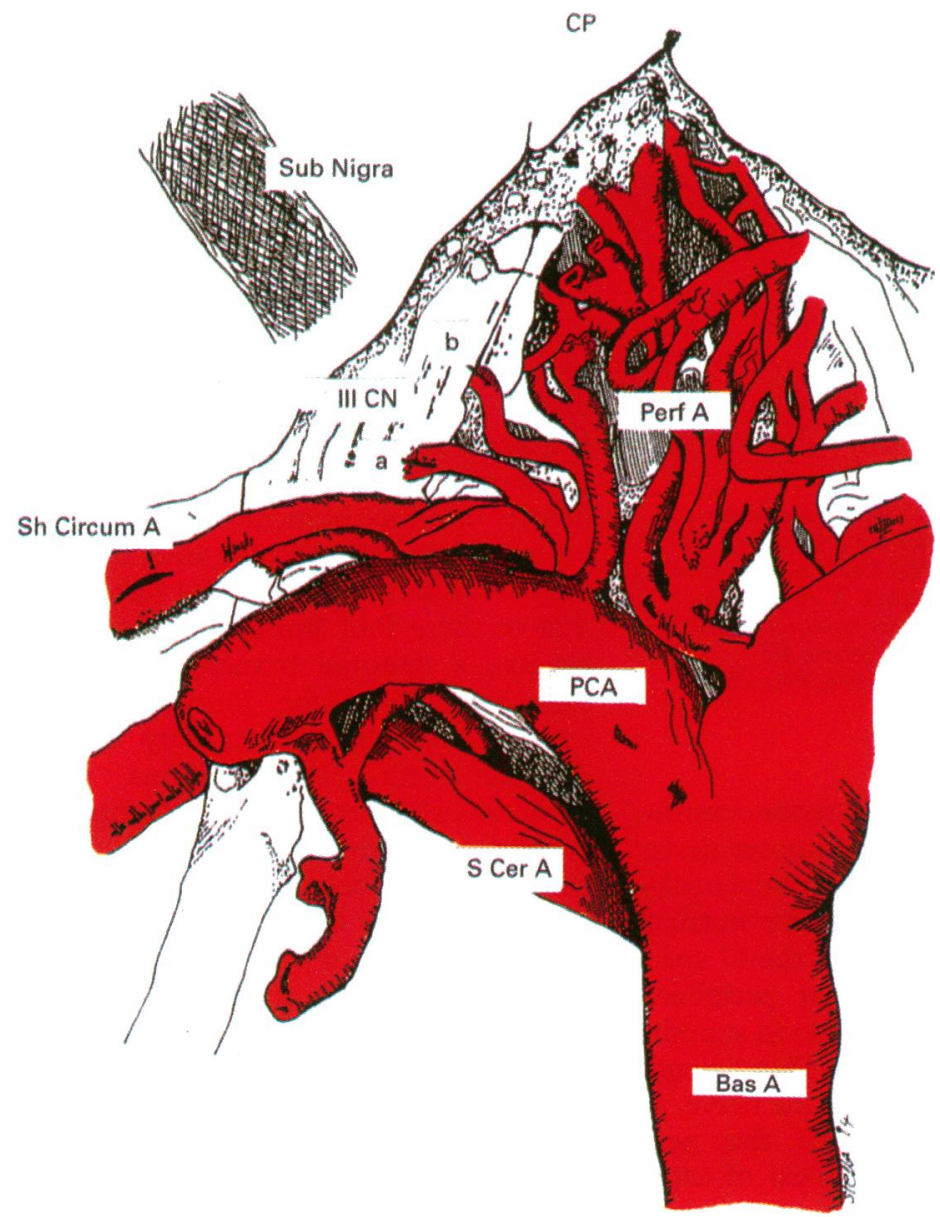

Fig $2 B$

Figure 2 (A) Photograph of proximal part of intracranial oculomotor nerve viewed from above. Demonstrates nerve being penetrated by thalamoperforating artery $(A)$ and being supplied by thalamoperforating vessels $(B)$. Magnification $\times 10$. (B) Drawing of specimen in $(A)$. Demonstrates proximal part of oculomotor nerve with thalamoperforating artery both penetrating and supplying the nerve. Bas $A=$ basilar artery, $S \mathrm{Cer} A=$ superior cerebellar artery, $P C A=$ posterior cerebral artery, $\operatorname{Perf} A=$ Thalamoperforating arteries, Sh Circum $A=$ short circumflex artery, $C P=$ cerebral peduncle, Sub Nigra=substantia nigra, III CN=oculomotor nerve, $a=$ thalamoperforating artery penetrating oculomotor nerve, $b=$ thalamoperforating artery supplying nutrient vessel to oculomotor nerve. oculomotor nerve using a serial section technique. However, this study also only commented that in general small nutrient arterioles from the posterior cerebral and basilar arteries supplied the proximal part of the intracranial oculomotor nerve.

Two more recent studies have demonstrated the proximal part of the intracranial oculomotor nerve to be supplied with extraneural nutrient arterioles arising from 'perforating vessels'. ${ }^{67}$ These are termed perforating vessels because they pierce the posterior perforated substance to supply various midbrain structures. The first of these undertaken by Pedroza et al, ${ }^{6}$ states that in $71 \%$ of specimens dissected non-specific perforating vessels vascularised the proximal part of the intracranial oculomotor nerve. The latter study by Marinkovic and Gibo, ${ }^{7}$ was more specific and was able to demonstrate nutrient arterioles arising from the mesencephalic perforators in $88.9 \%$ and/or the diencephalic perforators in $40.7 \%$ of cases. Furthermore, this latter study demonstrated that further extraneural nutrient vessels most commonly arose from the collicular artery (long circumflex artery) or branches.

Both Marinkovic and Gibo, ${ }^{7}$ and Milisvljevic et $a l^{8}$ have noted that the proximal part of the intracranial oculomotor nerve can be penetrated by either a perforating artery, a long or short circumflex artery, or both (56\% and $40 \%$ of cases respectively). While passing through the nerve these penetrating vessels give small extraneural nutrient arterioles to the proximal part of the nerve.

The present study utilised the anatomical details provided by the studies outlined above, ${ }^{2-8}$ as a springboard from which we started. In the specimens that we dissected only the thalamoperforating arteries were seen to provide nutrient arterioles to the proximal intracranial oculomotor nerve. The study undertaken by Marinkovic and $\mathrm{Gibo}^{7}$ differentiated the aforementioned perforating vessels into diencephalic perforators (thalamoperforating arteries) and mesencephalic perforators. Both of these groups supplied extraneural nutrient arterioles to the proximal intracranial oculomotor nerve as previously outlined. This difference between our findings and Marinkovic and Gibo's findings, ${ }^{7}$ could be accounted for by anatomical variation.

This study concurs with the findings of Marinkovic and Gibo, ${ }^{7}$ that other brainstem vessels (particularly the long circumflex artery) also provide extraneural nutrient arterioles to the proximal intracranial oculomotor nerve. Furthermore, we have also been able to confirm the findings of Marinkovic and $\mathrm{Gibo}^{7}$ and Milisavljevic et al, ${ }^{8}$ that one of the circumflex arteries or a thalamoperforating artery can penetrate the proximal part of the oculomotor nerve.

The extraneural blood supply to the middle part of the intracranial oculomotor nerve was more challenging. Neither Dreyfus et $a l^{2}$ nor Asbury et $a l^{5}$ specifically examined this part of the nerve. Common sense would have suggested before the study that a nutrient arteriole 


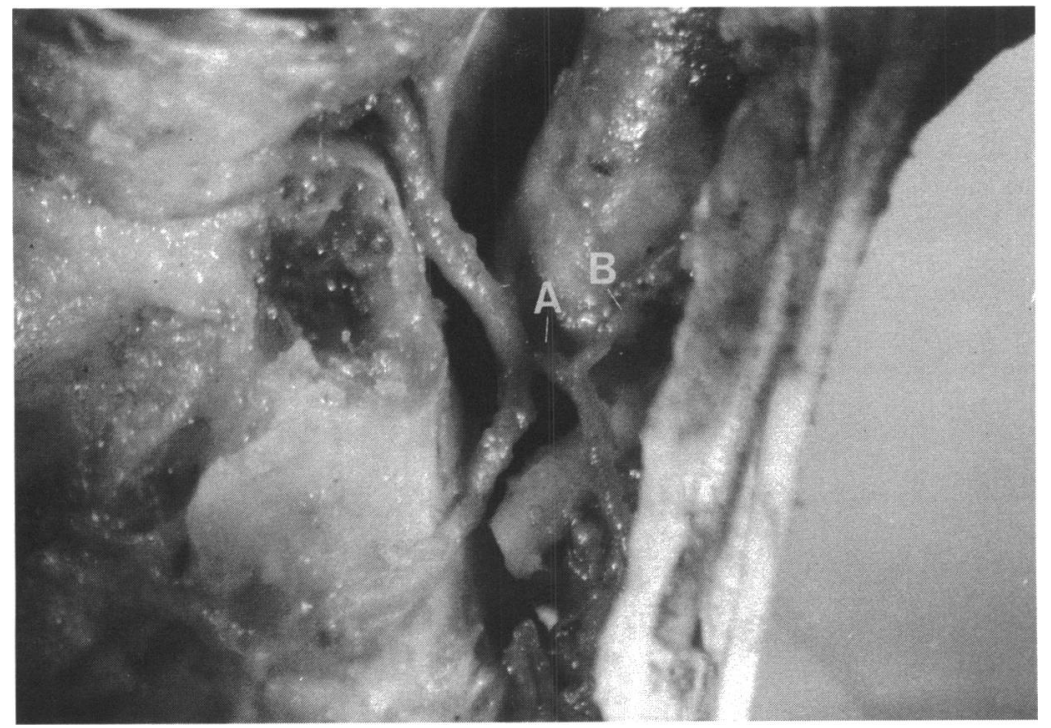

Fig $3 A$

B

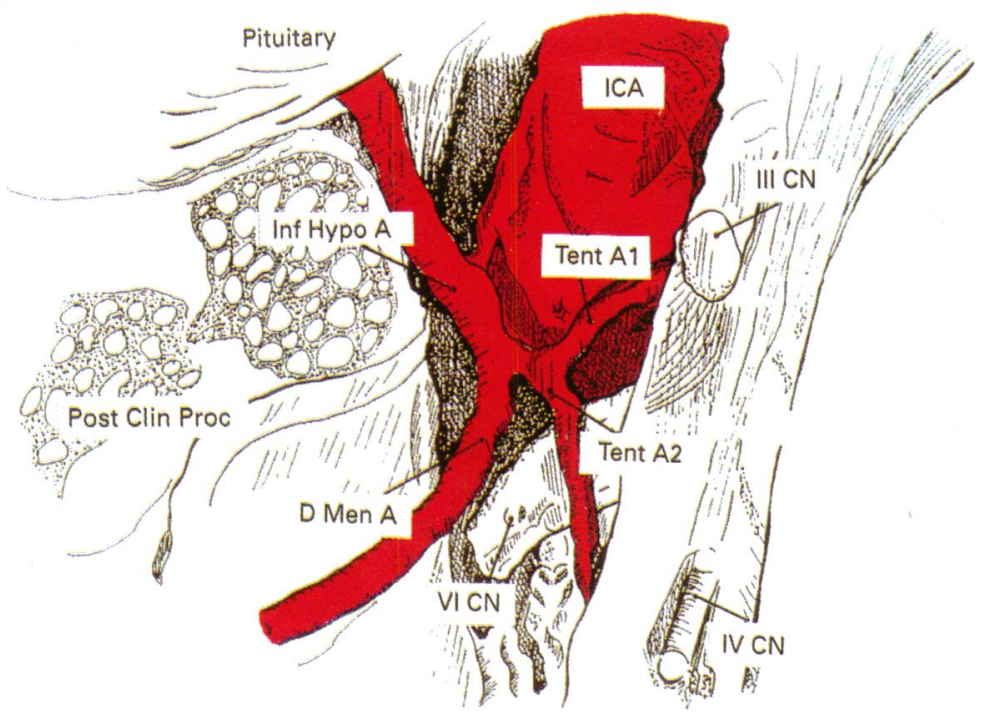

Fig 3B

Figure 3 (A) Photograph of right cavernous sinus viewed from above. Demonstrates meningohypophyseal trunk $(A)$ arising from the ICA. Note anterior tentorial artery $(B)$ supplying oculomotor nerve and tentorium. Magnification $\times 10$. (B) Drawing of specimen in $(A)$. Demonstrates meningohypophyseal trunk and its four branches. ICA=internal carotid artery, $D$ Men $A=$ dorsal meningeal artery, Tent $A 1=$ posterior tentorial artery, Tent $A 2=$ anterior tentorial artery, Inf Hypo $A=$ inferior hypophyseal artery, III $C N=$ oculomotor nerve, $I V C N=$ trochlear nerve, VI CN=abducens nerve, Post Clin Proc $=$ posterior clinoid process, Pituitary=pituitary gland.

would arise from the adjacent posterior communicating artery to supply the oculomotor nerve. In fact, the middle part of the intracranial oculomotor nerve was not seen to receive nutrient arterioles from any vessels in any of the $11 \mathrm{spec}-$ imens. Adams demonstrated that peripheral nerves have excellent intraneural blood supplies running within the epineurium. ${ }^{9}$ This extends from the proximal to distal parts of the nerve. At regular intervals branches pierce the perineurium and form longitudinal plexi of capillaries between nerve fascicles.

The present study did not examine the intraneural blood supply of the intracranial oculomotor nerve. However, we conclude that the absence of extraneural nutrient arterioles running onto the middle section of the oculomotor nerve suggests that this section of the nerve is supplied by intraneural arterioles passing from its proximal and distal ends.
The final division we deal with is the distal or intracavernous portion of the oculomotor nerve. Parkinson ${ }^{39}$ carried out a major anatomical study on over 200 cadavers in the early 1960s. In this landmark study, he attempted to name the branches of the intracavernous internal carotid artery by their distribution. In passing, Parkinson noted that a 'tentorial branch of the meningohypophyseal trunk' (Parkinson's nomenclature) supplied a nutrient arteriole to the oculomotor nerve. Parkinson named two other branches of the intracavernous internal carotid artery. He gave one the eponym 'McConnell's capsular artery' and the second the descriptive title 'the inferior cavernous sinus artery'. Parkinson did not comment as to whether or not either of these two branches supplied nutrient arterioles to the intracavernous oculomotor nerve. Asbury et $a l^{5}$ utilising Parkinson's nomenclature demonstrated that non-specific branches of the inferior cavernous sinus artery supplied nutrient arterioles to the intracavernous oculomotor nerve. Two further studies are noteworthy. ${ }^{112}$ The first one by Annabi et al, ${ }^{11}$ attempted to name the extraneural nutrient arterioles supplying the intracavernous oculomotor nerve but unfortunately they did not use Parkinson's nomenclature. The second study was a topographical anatomical study to find a more workable nomenclature for the branches of the intracavernous internal carotid artery, which does not comment on the blood supply to the oculomotor nerve. ${ }^{12}$

The present study utilises the nomenclature first outlined by Parkinson. ${ }^{3} 10$ Using clues from both Parkinson's ${ }^{3}$ and Asbury's work, ${ }^{5}$ we systematically examined the extraneural blood supply to the intracavernous oculomotor nerve. In our study reported in this paper, it has been demonstrated for the first time that the inferior cavernous sinus artery consistently divides into four branches. We have named the branches according to the directions in which they were seen to travel. We have also shown that of these four branches either an anterior branch, a tentorial branch, or both of these branches supply the intracavernous oculomotor nerve. In the majority of specimens dissected, the intracavernous oculomotor nerve was also supplied by the tentorial branch of the meningohypophyseal trunk. This branch had previously been demonstrated by Parkinson $^{310}$ to supply the oculomotor nerve.

Unfortunately it can be confusing if small arterioles are given long titles with similarly named branches. However, Parkinson's original nomenclature is that in common usage. Thus in this paper we have used the terms 'meningohypophyseal trunk' and 'the inferior cavernous sinus artery' and added the subdivision 'tentorial branch' to both these simply because it is a descriptive title and therefore more memorable. With regard to the extraneural blood supply to the intracavernous oculomotor nerve, we conclude that the arterioles arising from the inferior cavernous sinus artery are its main supply, as they are consistently present. The arterioles arising from the meningohypophyseal trunk (namely 


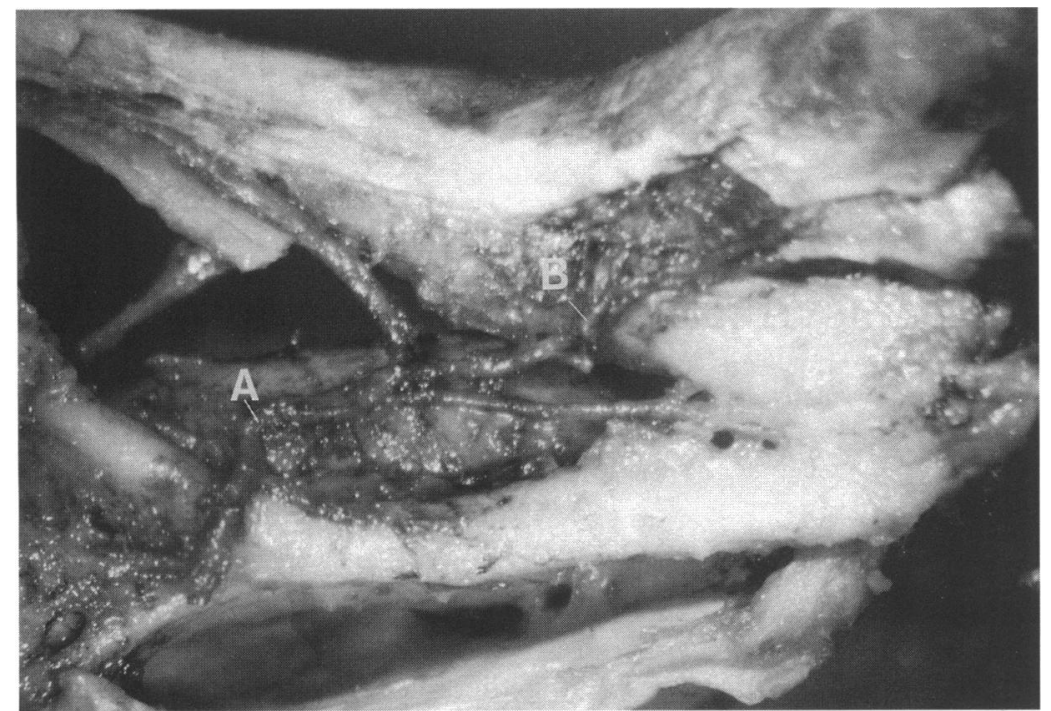

Fig $4 A$

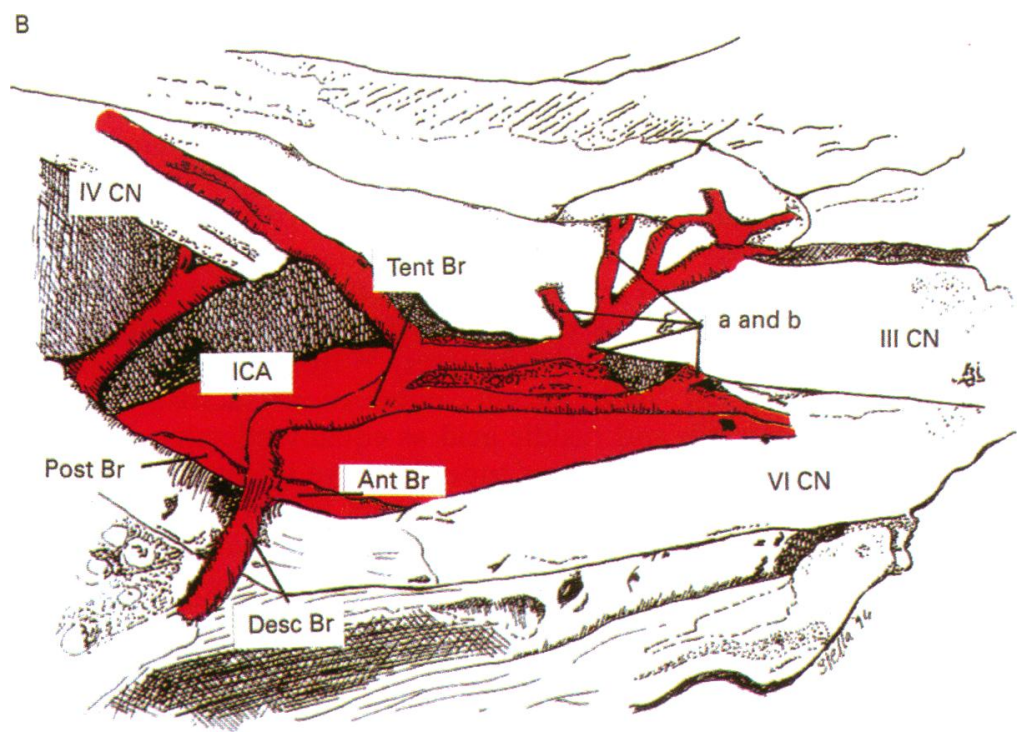

Fig $4 B$

Figure 4 (A) Photograph of right cavernous sinus viewed from the right side. Demonstrates the inferior cavernous sinus artery and its branches $(A)$. Note tentorial branch supplying oculomotor nerve $(B)$. Magnification $\times 10$. (B) Drawing of specimen in ( $A$ ). Demonstrates the four branches of the inferior cavernous sinus artery. ICA=internal carotid artery, Tent $\mathrm{Br}=$ tentorial branch, Ant $\mathrm{Br}=$ anterior branch, Post $\mathrm{Br}=$ posterior branch, Desc $B r=$ descending branch, $a$ and $b=$ branches of tentorial branches supplying oculomotor nerve, III CN=oculomotor nerve, IV CN=trochlear nerve, $V I C N=$ abducens nerve.

the anterior tentorial branch) provide supplementary blood supply to the oculomotor nerve.

Finally, we come to the relation between the blood supply to the pituitary gland and that to the intracavernous oculomotor nerve. A large part of the pituitary blood supply arises from the inferior hypophyseal artery, as determined by McConnell in $1958 .{ }^{4}$ The inferior hypophyseal artery is one of the three branches of the meningohypophyseal trunk, the same trunk which gives a tentorial branch to supply the oculomotor nerve. Thus we postulate that a vascular event occurring in this trunk could cause damage to both the pituitary gland and the oculomotor nerve.

\section{Conclusions}

A consistent pattern to the anatomy of the extraneural blood supply of the intracranial oculomotor nerve has been demonstrated. Proximally, the oculomotor nerve receives extraneural nutrient arterioles from the thalamoperforating arteries which are supplemented by other nutrient arterioles from brainstem vessels. The middle part of the intracranial oculomotor nerve does not receive extraneural nutrient arterioles from adjacent arteries. The distal (intracavernous) part of the intracranial oculomotor nerve receives extraneural nutrient arterioles from either the anterior branch, the tentorial branch, or both these branches of the inferior cavernous sinus artery. This may be supplemented by a tentorial branch of the meningohypophyseal trunk. There is a close relation between the blood supply to the pituitary gland and the intracavernous oculomotor nerve - namely, the shared meningohypophyseal trunk.

1 Bartholdy K. Die Arterien der Nerven. Morphol Arb 1897; 7 ; 393-458

2 Dreyfus PM, Hakin S, Adams RD. Diabetic ophthalmoplegia. AMA Arch Neurol Psychiatry 1957; 77: 337-49.

Parkinson D. A surgical approach to the cavernous portion Parkinson D. A surgical approach to the cavernous port
of the carotid artery. $\mathcal{F}$ Neurosurg $1965 ; 23: 474-83$.

4 McConnell EM. The arterial blood supply of human hypophysis cerebri. Anat Rec 1953; 115: 175-201.

5 Asbury AK, Aldredge N, Hershberg R, Fisher CM. Oculomotor palsy in diabetes mellitus: a clinico-pathological study. Brain 1970; 93: 555-66.

6 Pedroza A, Dujovny M, Ausman JI, Diaz FG, Cabezudo Artero J, Berman SK, et al. Microvascular anatomy of the interpeduncular fossa. $\mathcal{F}$ Neurosurg 1986; 64: 484-93.

7 Marinkovic S, Gibo $H$. The neurovascular relationship and the blood supply of the oculomotor nerve: the microsurgical anatomy of its cisternal segment. Surg Neurol surgical anatomy

8 Milisavljevic $M$, Marinkovic $S$, Lolic Draganic V, Kovacevic $M$. Oculomotor, trochlear and abducens nerves penetrated by cerebral vessels. Arch Neurol 1986; 43: 505-16.

9 Adams WE. The blood supply of nerves II. The effects of exclusion of its regional sources of supply on the sciatic nerve of the rabbit. $\mathcal{F}$ Anat 1943; 77: 243-50.

10 Parkinson D. Collateral circulation of cavernous carotid artery: anatomy. Can f Surg 1964; 7: 251-68.

11 Annabi A, Lasjaunias P, Lapresle J. Paralysies de la IIIe paire au cours du diabete et vascularisation du moteur paire au cours du diabete et vascularisation du

12 Tran-Dinh H. Cavernous branches of the internal carotid artery: anatomy and nomenclature. Neurosurgery 1987; 20: 205-10. 